\title{
Diseño y Construcción de una Escala de Conducta Prosocial para Adultos
}

\author{
Design and Construction of a Prosocial Behavior Scale for Adults
}

\author{
Sofía Esmeralda Auné ${ }^{1}$, Facundo Juan Pablo $\mathrm{Abal}^{2}$ y Horacio Félix Attorresi ${ }^{3}$
}

\begin{abstract}
Resumen
Se presenta el proceso de construcción de una escala de Comportamiento Prosocial para adultos. Para ello, se realizaron análisis de confiabilidad y dimensionalidad desde el marco de la Teoría Clásica de Tests. Se evaluaron 90 ítems con el método de jueces expertos, luego los ítems conservados fueron administrados en grupos reducidos. Participaron 576 estudiantes de Psicología de la Universidad de Buenos Aires. El análisis de la consistencia interna brindó un índice satisfactorio $(\alpha=.85$; glb=.93), mientras que el estudio de la dimensionalidad mostró una estructura de cuatro factores: Comportamientos Empáticos, Altruismo, Ayuda y Compartir. Numerosas conductas vinculadas con el refuerzo y soporte emocional exhibieron un promedio alto, mientras que se observó un bajo puntaje en los ítems que reflejan los comportamientos típicamente benéficos, tales como voluntariado, donación de ropa, comida o dinero y en reactivos que expresan comportamientos altruistas extremos.
\end{abstract}

Palabras clave: conducta prosocial, altruismo, ayuda, compartir

\begin{abstract}
The process of constructing a scale of Prosocial Behavior for adults is presented. Reliability and dimensionality tests were conducted from the Classical Test Theory perspective. 90 items were assessed by means of the expect judge method; the items shortlisted were administered to reduced groups. 576 students of Psychology of the University of Buenos Aires, took part in the test. The internal consistency analysis shows a satisfactory ratio $(\alpha=.85 ; \mathrm{glb}=.93)$, while the dimensionality analysis presents a four-factor structure. These factors were Empathic Behavior, Altruism, Help and Share.Numerous behaviors connected with emotional reinforcement and support posted a high average, while the items typically associated with philanthropist behavior, such as volunteer work, clothes/food/money donations, and with highly ultraistic practices yielded low scores.
\end{abstract}

Keywords: prosocial behavior, altruism, help, share

La investigación que se presenta en este artículo fue realizada con subsidios de la Universidad de Buenos Aires UBACyT 2014-17 Código Nº 20020130100320BA y de la Agencia Nacional de Promoción Científica y Tecnológica ANPCyT PICT 2011-0826.

${ }^{1}$ Lic. y Prof. en Psicología. Facultad de Psicología, Universidad de Buenos Aires, Argentina. Ayudante de Trabajos Prácticos. Dirección postal: Virrey Liniers 577 15 B (1220), CABA. Teléfono: 005411-49313374. E-mail: sofiaaune@psi.uba.ar

${ }^{2}$ Dr. en Psicología. Facultad de Psicología, Universidad de Buenos Aires, Argentina. Jefe de Trabajos Prácticos. Zuviría 5691 (1439), CABA. Teléfono: 005411-46014331. E-mail: fabal@psi.uba.ar

${ }^{3}$ Lic. en Ciencias Matemáticas. Facultad de Psicología, Universidad de Buenos Aires, Argentina. Profesor Titular Regular. Rivera Indarte 132, $1^{\circ}$ A (1406), CABA. Teléfono: 005411-46370923. E-mail: hattorre@psi.uba.ar 


\section{Introducción}

Los comportamientos prosociales son definidos como conductas voluntarias orientadas a beneficiar a otros (Caprara, Steca, Zelli, \& Capanna, 2005) que pueden realizarse con o sin motivación altruista (González Portal, 2000). Acciones de este tipo tales como compartir, donar, cuidar, confortar y ayudar son consideradas beneficiosas para las relaciones sociales y el bienestar personal (Alessandri, Caprara, Eisenberg, \& Steca, 2009; Eisenberg, Fabes, \& Spinrad, 2006). La Conducta Prosocial favorece la aceptación de los pares y la formación de relaciones interpersonales positivas (Eisenberg et al., 2006), oponiéndose a la Conducta Antisocial, que incluye aquellos comportamientos dañinos para la sociedad (Luengo, Otero, GómezFraguela, \& Tavares-Filho, 1999).

Según González Portal (2000), se considera que la tendencia a realizar conductas prosociales es dependiente principalmente de la situación, del tipo de Conducta Prosocial evaluada y de las variables de personalidad implicadas. En este sentido, algunas personas se inclinan más que otras a realizar comportamientos que benefician a otros. Se postula que esos comportamientos pertenecen a una dimensión latente y relativamente estable de la personalidad llamada prosocialidad (Caprara, Alessandri, Di Giunta, Panerai, \& Eisenberg, 2010). Los valores y las creencias de autoeficacia contribuyen a formar esta tendencia (Alessandri et al., 2009). Los valores definen los objetivos a perseguir mientras que las creencias de autoeficacia se relacionan con la confianza en la propia capacidad para alcanzar dichas metas. Los valores de autotrascendencia contribuyen a la creación de una mentalidad favorable a las emociones y conductas asociadas con la empatía y la acción prosocial (Alessandri et al., 2009; Caprara, Alessandri, \& Eisenberg, 2012). Otros factores que favorecen la Conducta Prosocial son el control emocional y la capacidad de toma de perspectiva (Alessandri et al., 2009).

La motivación de la Conducta Prosocial es frecuentemente difícil de determinar, según se desprende de investigaciones previas acerca del altruismo (Abal, Lozzia, Blum, Galibert, Aguerri, \& Attorresi, 2010; Attorresi, Abal, Lozzia, Picón
Janeiro, Aguerri, \& Galibert, 2008). Puede incluir el deseo de aprobación o de actuar en concordancia con determinados valores morales (Batson \& Powell, 2003). La empatía y la simpatía son importantes factores motivadores del comportamiento prosocial. Por el contrario, las reacciones emocionales autocentradas tienden a socavarlo, aunque podrían derivar en un comportamiento de ayuda cuando éste es un medio para lograr el beneficio personal (Batson \& Powell, 2003; Eisenberg, Cumberland, Guthrie, Murphy, \& Shepard, 2005).

La Conducta Prosocial ha sido estudiada principalmente en niños y adolescentes. Para este rango etario se ha descubierto, por ejemplo, que el aprendizaje y el logro son mayores en los alumnos adolescentes prosociales (Inglés, MartínezGonzález, García-Fernández, \& Ruiz-Esteban, 2010). La mayoría de los instrumentos de evaluación se centran en estas etapas. En cuanto a su evaluación en adultos la Conducta Prosocial se valora mediante Escalas de Autoinforme, dado que se supone una mayor capacidad de autoobservación que en el caso de los adolescentes y de los niños, donde se apela frecuentemente a un espectador diferente al propio sujeto (Auné, Blum, Abal, Lozzia, \& Attorresi, 2014). La construcción de test para medir Conducta Prosocial en la adultez es relativamente tardía con respecto a la creación de instrumentos para la medición en etapas previas (Auné et al., 2014; Caprara et al., 2005). Sin embargo, es particularmente relevante dado que este tipo de conducta favorece el bienestar personal y social (Caprara et al., 2012). Existen además ciertas conductas prosociales que sólo son realizables desde la etapa adulta, donde la prosocialidad adquiere características propias (Capraraet al., 2005). Incluso, conductas consideradascomo prosociales a ciertaedad, pueden no serlo en otra (Bravo \& Lagunes, 2009). En dicho contexto, surge la necesidad de contar con nuevos instrumentos, específicos para dicho período vital.

Se realizó una revisión de las tipologías más recientes de Conducta Prosocialcon el fin de definir adecuadamente el constructo (Caprara et al., 2005; Hay \& Cook, 2007; López de Cózar, Esteban Martínez, Calatayud Miñana, \& Alamar Rocati, 2008; Warneken \& Tomasello, 2009), 
observándose las dimensiones más frecuentes y relevantes postuladas en la literatura específica (Auné et al., 2014). Dicha labor permitió delimitar una clasificación de cuatro dimensiones: "Comportamientos Empáticos" (CE), "Altruismo" (AL), "Ayuda" (AY) y "Compartir y Donar" (CD). Se definió operacionalmente Comportamientos Empáticos como aquellos comportamientos que manifiestan empatía, comprensión, refuerzo y soporte emocional. Se consideró como Altruistas a aquellas conductas que impliquen beneficiar a otros, aunque esto pueda perjudicar el bienestar personal. La dimensión de Ayuda se operacionalizó como comportamientos de asistencia, cuidado y compromiso con los otros. Implica necesariamente un benefactor y un beneficiario claramente diferenciados. Por último, se entendió por Compartir y Donar: dar, donar, prestar o compartir objetos, tiempo, dinero, experiencias u otro aspecto de valor para los usos y costumbres (Auné et al., 2014).

A la luz de estas consideraciones teóricas y técnicas, se plantea como objetivo general de este trabajo presentar las características psicométricas de un autoinforme original para medir Conducta Prosocial en adultos, elaborado en el marco de la Teoría Clásica de Tests. La investigación se ajusta a las pautas éticas propuestas por American Educational Research Association, American Psychological Association, \& National Council on Measurement in Education (1999).

\section{Método}

\section{Participantes}

Se solicitó la colaboración voluntaria de estudiantes del segundo año de la carrera de Licenciatura en Psicología de la Universidad de Buenos Aires (UBA) de Argentina. Para la selección de los individuos se aplicó un diseño muestral no probabilístico por accesibilidad o conveniencia (Gil-Escudero y Martínez-Arias, 2001). La muestra estuvo compuesta por 576 sujetos, de los cuales $81 \%$ fueron mujeres y $19 \%$ varones. La edad varió entre 18 y 58 años con una media de 22.5 , una mediana de 21 y un desvío estándar de 5.35.

\section{Instrumento}

Escala de Conducta Prosocial [ECP]. Se elaboró un conjunto inicial de 90 ítems representativos de las cuatro dimensiones más importantes identificadas en un estudio previo (Auné et al., 2014): Comportamientos Empáticos (CE), Altruismo (AL), Ayuda (AY) y Compartir y Donar (CD). Cada reactivo hizo referencia a un Comportamiento Prosocial específico. Por ejemplo, algunos de los ítems construidos fueron: Cuando siento que alguien está mal, le demuestro que lo entiendo (CE), Aunque pudiera cobrar mucho dinero por él, compartiría gratuitamente un descubrimiento que contribuyera a un mundo mejor (AL), Ayudo a ancianos a cruzar la calle o cargar objetos (AY), Comparto las malas experiencias de la vida con amigos para que no cometan los mismos errores (CD). En el caso de que el sujeto no haya tenido la oportunidad de estar en una situación que requiera la conducta se aclaró que debe responder en base a cómo creería que actuaría, con sus valores, personalidad y mentalidad, imaginándose la situación. Se pidió expresamente sinceridad al responder, explicando que no hay respuestas correctas o incorrectas y que la prueba es anónima. El anonimato tendería a disminuir la deseabilidad social (Fisher, 1993).

Se procuró que estos 90 ítems abarcaran distintas intensidades de beneficio hacia el otro, así como diferentes receptores de la acción (compañeros, amigos, conocidos, desconocidos). Se buscó que fueran claros, de fácil comprensión, relevantes y representativos de cada categoría. Los ítems se redactaron en dos direcciones para prevenir la tendencia a elegir las opciones favorables - aquiescencia - y con contenidos que discriminan en todos los niveles de Conducta Prosocial incluidos los extremos. Además, se cuidó de redactar una cantidad equilibrada de reactivos por cada dimensión.

La modalidad de respuesta se especificó con una escala Likert de seis opciones que refleja la frecuencia con que se realiza la acción (1=Nunca, 2=Casi Nunca, 3=A Veces, 4=Con Frecuencia, $5=$ Casi Siempre, $6=$ Siempre). No se ha incluido una categoría central.

Se planteó al evaluado un conjunto de situaciones habituales con el fin de obtener información acerca de cómo se comporta 
usualmente, lo que es característico de los tests de comportamiento típico (Cronbach, 1972). Se han utilizado, entonces, en los ítems, conductas generales propias de la vida cotidiana (e.g. Intento "levantar" la autoestima a mis amigos) así como comportamientos específicos del contexto universitario (e.g. Comparto con mis compañeros las experiencias que tuve en las materias que ya cursé). En ningún caso se incluyó en el ítem la motivación con la cual se realizó la conducta, ya que el concepto de Comportamiento Prosocial pone el acento en la acción en sí misma es decir, en el acto de beneficiar a otros, sea la intención primaria altruista o egoísta.

\section{Procedimiento}

Los reactivos fueron sometidos a un proceso de depuración primaria. En primer lugar, fueron evaluados por cuatro jueces expertos en la construcción de test de comportamiento típico en base a los siguientes criterios: a) Congruencia con la definición operacional de la dimensión medida, b) Grado de relevancia como indicador del constructo, c) Adecuación respecto de los aspectos sintácticos y semánticos y al nivel de comprensión de la población muestreada (Cohen \& Swerdlik, 2006; Tornimbeni, Pérez, \& Olaz, 2008). El análisis de los conceptos emitidos por los jueces se realizó siguiendo los criterios de Compas, Davis, Forsythe, \& Wagner (1987) es decir el porcentaje de acuerdo entre jueces.

Posteriormente, se realizó una prueba piloto con los reactivos conservados en un grupo de 32 sujetos con el fin de detectar aquellos términos o expresiones que resulten confusos para los examinados tanto en los ítems como en las instrucciones o perjudiquen la validez aparente de la prueba. Incorporadas las modificaciones, el protocolo se administró nuevamente, sin tiempo límite, a veinticuatro grupos con un máximo de 35 personas cada uno. Además de ser la prueba anónima, se informó a los participantes que los datos derivados de esta investigación se utilizarán con fines exclusivamente científicos. También se pidió el consentimiento informado a cada uno de los sujetos.

Las respuestas fueron volcadas en una base de datos aplicando el formato de codificación tradicional. Se homogeneizó el sentido de los ítems para que en todos los reactivos las opciones se encuentren ordenadas de menor a mayor nivel del constructo medido. Es decir, por ejemplo, que responder "Siempre" en un ítem redactado en sentido positivo equivale a responder "Nunca" a un ítem de sentido negativo.

A continuación se obtuvieron distribuciones de frecuencias, estadísticos descriptivos de cada ítem: media (M), mediana (Mdn), desvío estándar (S), asimetría (As) y curtosis (K). Para evaluar la confiabilidad se calculó el Alfa de Cronbach con el Statistical Package for Social Sciences (SPSS) versión 18 y el Greatest Lower Bound (glb) con el programa Factor 9.2 (Lorenzo-Seva \& Ferrando, 2006).

Se descartaron los ítems que a) su eliminación aumenta el Alfa de Cronbach de su factor, y/o b) mostraban una saturación factorial de la solución sin rotar con su factor de mayor carga menor a .35 (Cohen \& Manion, 2002), y/o c) presentaran doble pesaje y/o d) poseían un índice de asimetría o curtosis en valor absoluto mayor a 1 (George \& Mallery, 2001).

Con el fin de estudiar la dimensionalidad, se realizó un análisis factorial exploratorio con la matriz de correlaciones policóricas (Brown, 2006) teniendo en cuenta la naturaleza politómica de las respuestas (García-Cueto \& Fidalgo, 2005) mediante los programas Microfact 2.0 (Waller, 2001) y Factor 9.2 (Lorenzo-Seva \& Ferrando, 2006). Se aplicó como procedimiento para la extracción de factores el método de mínimos cuadrados simple sobre dicha matriz de correlaciones policóricas. Se tomó además el Criterio de caída de Cattell(1966, citado en García-Cueto \& Fidalgo, 2005) para considerar la dimensionalidad de la Prueba. Es decir, se establecieron tantas dimensiones como saltos bruscos en el diagrama de autovalores hasta mostrar el paralelismo con el eje de abscisas (García-Cueto \& Fidalgo, 2005).

Asimismo, cada factor debió poseer al menos 4 ítems con correlaciones iguales o superiores a .40, para considerarlo como tal. Se interpretó al factor atendiendo a las correlaciones ítem-factor más elevadas (Glutting, Monaghan, Adams, \& Sheslow, 2002). 


\section{Resultados}

\section{Crítica de jueces expertos}

El porcentaje de acuerdo interjueces destaca la pertinencia de la adaptación lingüística pues alcanzó un $70 \%$ de concordancia entre los evaluadores en su primera revisión, finalizando con un $90 \%$ posterior una vez realizadas las sugerencias indicadas.

Un total de 62 ítems fueron los que cumplieron con los criterios luego de la depuración por jueces. Por ejemplo, un ítem descartado principalmente en base al criterio c fue "pongo una barrera entre mi persona y los problemas ajenos" (CE- redactado en sentido negativo). Los jueces destacaron confusión en la interpretación en lo que significa "poner una barrera" ya que puede significar, en un sentido, desentenderse o, en otro, no verse afectado para poder así ayudar. Los jueces expertos también evaluaron las consignas y las opciones de respuesta, aportando sugerencias y modificaciones.

\section{Estadísticos descriptivos}

Descriptivamente, los ítems que exhibieron puntuaciones más altas fueron: Felicito a quienes se esfuerzan para conseguir sus metas ( $\mathrm{M}=5.3$; $\mathrm{Mdn}=6)$, Me satisface ver los defectos ajenos $(\mathrm{M}=5.2 ; \mathrm{Mdn}=6)$, Escucho con atención al que necesita contar sus problemas ( $\mathrm{M}=5.1$; Mdn=5),Acompaño a conocidos en momentos dolorosos ( $\mathrm{M}=4.9 ; \quad \mathrm{Mdn}=5)$ y Me molesta escuchar a un amigo cuando se pone insistente con sus problemas $(\mathrm{M}=4.8 ; \mathrm{Mdn}=5)$.

En el otro extremo, los ítems que recibieron puntuaciones más bajas fueron: Me quedo con lo justo y necesario para vivir, y reparto todo lo demás $(\mathrm{M}=2.2 ; \mathrm{Mdn}=2)$, Dono dinero o comida a comedores comunitarios $(\mathrm{M}=2.2 ; \mathrm{Mdn}=2)$, Hago donaciones a organizaciones benéficas $(\mathrm{M}=2.4$; $\mathrm{Mdn}=2)$, Si puedo hacerlo, dono sangre para desconocidos $(\mathrm{M}=2.3 ; \quad \mathrm{Mdn}=2), \quad$ Realizo actividades como voluntario $(\mathrm{M}=2.3 ; \mathrm{Mdn}=2) \mathrm{y}$ Presto objetos de valor a cualquiera ( $\mathrm{M}=2.4$; $\mathrm{Mdn}=2)$. En ítem Realizo actividades como voluntario, por ejemplo, el $64.2 \%$ de los sujetos se concentra en las categorías Nunca y Casi Nunca, las categorías A veces y Con Frecuencia reúnen $30 \%$ de los casos, mientras que sólo 5,8 \% manifiesta que Casi Siempre o Siempre lo hace.

Los ítems que mostraron un comportamiento más variable entre los sujetos fueron: Realizo trámites y pagos para una persona anciana o con dificultades físicas $(\mathrm{S}=1.5)$, Aunque pudiera cobrar mucho dinero por él, compartiría gratuitamente un descubrimiento que contribuyera a un mundo mejor $(\mathrm{S}=1.5)$. Si tuviera un único plato de comida, lo compartiría con un desconocido, Apoyo los paros por causas que me parecen justas aunque los mismos me perjudiquen ( $\mathrm{S}=1.4)$ y Si puedo hacerlo, dono sangre para desconocidos $(\mathrm{S}=1.4)$.

Los ítems que, por el contrario, mostraron un comportamiento más homogéneo fueron: $\mathrm{Me}$ desentiendo de los problemas ajenos $(\mathrm{S}=.8)$, Escucho con atención al que necesita contar sus problemas $(\mathrm{S}=.8)$, Digo mentiras $(\mathrm{S}=.9)$, Felicito a quienes se esfuerzan para conseguir sus metas $(\mathrm{S}=.9)$, Evito prestar mis objetos a amigos $(\mathrm{S}=.9)$ y Me molesta cuando mis amigos intentan cargarme con sus problemas.

\section{Estudio de confiabilidad}

El Alfa de Cronbach resultó alto $(\alpha=.85)$. Sin embargo, este resultado tiene una interpretación limitada en cuanto a la consistencia interna ya que existen actualmente métodos más potentes en este sentido, como el glb. El glb fue de .93. Tanto el Alfa de Cronbach como el glb se basan en la matriz de varianzas y covarianzas de los ítems del test (Bendermacher, 2010) y han arrojado resultados satisfactorios. El análisis de confiabilidad de cada subescala se realizó sólo con los ítems que mostraban una saturación factorial con su factor de mayor carga mayor a .35 y se presenta en la Tabla 2.

Siguiendo a DeVellis (2003) y a Gempp, Denegri, Caripán, Catalán, Hermosilla y Caprile (2007) las cuatrosubescalas presentaron un nivel adecuado de confiabilidad.

Se puede observar en la Tabla 3 la existencia de una correlación baja a moderada y positiva entre las subescalas, siendo la más fuerte (.42) entre Ayuda y Altruismo. 
Tabla 1. Estadísticos descriptivos por ítem

1. Me alejo de los que están mal.

1. Me alejo de los que están mal.

2. Hago donaciones a organizaciones benéficas.

3. Apoyo los paros por causas que me parecen justas aunque los mismos me perjudiquen.

4. Escucho con atención al que necesita contar sus problemas.

5. Me quedo con lo justo y necesario para vivir, y reparto todo lo demás.

6. Comparto las malas experiencias de la vida con amigos para que no cometan los mismos errores.

7. Hago grandes sacrificios sin recibir nada a cambio.

8. Me satisface ver los defectos ajenos.

9. Destaco a quienes hacen las cosas bien.

10. Digo mentiras.

11. Participo en actividades solidarias.

12. Me pongo en el lugar del otro.

13. Comparto con mis compañeros las experiencias que tuve en las materias que ya cursé

14. Ayudo a los conocidos a enfrentar sus problemas.

15. Intento "levantar" la autoestima a mis amigos.

16. Si una persona me cuenta un conflicto, intento que comprenda el punto de vista de la otra parte.

17. Reconforto en momentos dolorosos a personas que conozco poco.

18. Si mis compañeros están con dificultades, no me molesta hacer tareas de más en un trabajo de grupo.

19. Dedico un tiempo importante de mi vida a mejorar el mundo.

20. Expreso abiertamente mi apoyo a minorías discriminadas aunque no pertenezca a las mismas.

21. Evito involucrarme demasiado en los problemas ajenos.

22. Les digo a los demás lo mucho que valen.

23. Presto objetos de valor a cualquiera.

24. Me molesta escuchar a un amigo cuando se pone insistente con sus problemas.

25. Aunque lo podría vender, dono lo que no uso o no me sirve.

26. Si entiendo un tema, lo explico a mis compañeros con gusto.

27. Ofrezco dinero a mis amigos cuando pienso que lo necesitan.

28. Elogio a quien se lo merece.

29. No me arriesgo para defender a alguien que corre peligro.

30. Siento todo dolor ajeno como propio.

31. Realizo trámites y pagos para una persona anciana o con dificultades físicas.

32. Evito a los desconocidos que empiezan a comentarme sus problemas.

33. Aunque pudiera cobrar mucho dinero por él, compartiría gratuitamente un descubrimiento que contribuyera a un mundo mejor.

34. Felicito a quienes se esfuerzan para conseguir sus metas.

35. Dono dinero o comida a comedores comunitarios.

36. Me ocupo sólo de mí.

37. Comparto mis conocimientos con compañeros.

38. Intervengo a favor de cualquiera que esté siendo tratado injustamente.

39. Realizo actividades como voluntario.

40. Actúo como bastón de los demás.

41. Evito prestar mis objetos a amigos.

42. Ayudo a los conocidos a enfrentar situaciones conflictivas o estresantes.

43. Si tuviera un único plato de comida, lo compartiría con un desconocido.

44. Apoyo los reclamos justos aunque me perjudiquen de forma significativa.

45. Acompaño a conocidos en momentos dolorosos.

46. Explico a mis compañeros cómo hacer los trámites de la facultad.

47. Intento mejorar la autoestima de mis amigos.

48. No me comprometo en la defensa de alguien que está siendo atacado.

49. Doy hasta lo que no tengo.

50. A pesar de que no me guste, si es necesario hago tareas de más.

51. Presto apuntes o libros a compañeros.

52. Ayudo a ancianos a cruzar la calle o cargar objetos.

53. Cuando siento que alguien está mal, le demuestro que lo entiendo.

54. Doy dinero a cualquiera que lo necesite.

55. Resalto los defectos de los demás.

56. Me molesta cuando mis amigos intentan cargarme con sus problemas.

57. Intento que las personas en conflicto comprendan el punto de vista de la otra parte.

58. Si puedo hacerlo, dono sangre para desconocidos.

59. No miento.

60. Me desentiendo de los problemas ajenos.

61. Me ocupo del bienestar de cualquier individuo, grupo o comunidad.

62. Presto dinero a mis amigos aún sabiendo que quizás no puedan devolvérmelo.

$\begin{array}{llll}\text { M } & \text { S } & \text { As } & \text { K } \\ 4.7 & 1.04 & -0.9 & 1.1\end{array}$

$\begin{array}{llll}2.5 & 1.23 & 0.6 & 0.0\end{array}$

$\begin{array}{llll}2.8 & 1.45 & 0.5 & -0.5\end{array}$

$\begin{array}{llll}5.0 & 0.88 & -0.7 & -0.3\end{array}$

$\begin{array}{llll}2.2 & 1.05 & 0.7 & 0.4\end{array}$

$\begin{array}{llll}3.9 & 1.28 & 0.0 & -0.9\end{array}$

$\begin{array}{llll}3.1 & 1.11 & 0.3 & -0.1\end{array}$

$\begin{array}{llll}5.2 & 1.00 & -1.7 & 4.0\end{array}$

$\begin{array}{llll}4.4 & 1.04 & -0.2 & -0.4\end{array}$

$\begin{array}{llll}4.4 & 0.89 & -0.8 & 2.2\end{array}$

$\begin{array}{rrrr}2.7 & 1.20 & 0.8 & 0.7\end{array}$

$\begin{array}{llll}4.3 & 1.06 & -0.2 & -0.5\end{array}$

$\begin{array}{llll}4.7 & 1.19 & -0.7 & -0.2\end{array}$

$\begin{array}{llll}4.6 & 1.10 & -0.4 & -0.7\end{array}$

$\begin{array}{cccc}5.0 & 1.01 & -1.0 & 0.7\end{array}$

$\begin{array}{llll}4.5 & 1.03 & -0.2 & -1.1\end{array}$

$\begin{array}{llll}3.5 & 1.20 & 0.2 & -0.3\end{array}$

$\begin{array}{llll}3.6 & 1.28 & 0.2 & -0.6\end{array}$

$\begin{array}{rrrr}2.7 & 1.06 & 0.7 & 0.3\end{array}$

$\begin{array}{llll}3.8 & 1.37 & -0.1 & -0.8\end{array}$

$\begin{array}{llll}3.7 & 1.16 & -0.5 & 0.0\end{array}$

$\begin{array}{llll}4.0 & 1.18 & -0.1 & -0.5\end{array}$

$\begin{array}{llll}2.4 & 1.10 & 0.9 & 0.5\end{array}$

$\begin{array}{llll}4.8 & 0.97 & -0.8 & 0.8\end{array}$

$\begin{array}{llll}4.1 & 1.46 & -0.4 & -0.8\end{array}$

$\begin{array}{llll}4.7 & 1.15 & -0.6 & -0.5\end{array}$

$\begin{array}{llll}4.0 & 1.35 & -0.1 & -1.0\end{array}$

$\begin{array}{llll}4.5 & 1.13 & -0.4 & -0.7\end{array}$

$\begin{array}{llll}4.2 & 1.16 & -0.9 & 0.9\end{array}$

$\begin{array}{llll}3.0 & 1.12 & 0.3 & -0.2\end{array}$

$\begin{array}{llll}2.7 & 1.44 & 0.6 & -0.3\end{array}$

$\begin{array}{llll}4.1 & 1.21 & -0.6 & 0.0\end{array}$

$\begin{array}{llll}4.0 & 1.49 & -0.2 & -1.0\end{array}$

$\begin{array}{lllll}5.3 & 0.92 & -1.4 & 1.3\end{array}$

$\begin{array}{llll}2,2 & 1.16 & 0.8 & 0.3\end{array}$

$\begin{array}{llll}4.5 & 1.09 & -0.5 & 0.0\end{array}$

$\begin{array}{llll}4.4 & 1.02 & 0.0 & -0.9\end{array}$

$\begin{array}{llll}3.9 & 1.15 & 0.1 & -1.0\end{array}$

$\begin{array}{llll}2.3 & 1.24 & 1.0 & 0.8\end{array}$

$\begin{array}{llll}3.2 & 1.02 & 0.3 & 0.3\end{array}$

$\begin{array}{llll}5.0 & 0.91 & -1.1 & 1.7\end{array}$

$\begin{array}{llll}4.2 & 1.09 & -0.2 & -0.7\end{array}$

$\begin{array}{llll}3.6 & 1.44 & 0.0 & -1.0\end{array}$

$\begin{array}{llll}3.7 & 1.28 & 0.2 & -0.7\end{array}$

$\begin{array}{llll}4.9 & 1.14 & -1.0 & 0.5\end{array}$

$\begin{array}{llll}4.6 & 1.27 & -0.6 & -0.4\end{array}$

$\begin{array}{llll}4.7 & 1.09 & -0.5 & -0.6\end{array}$

$\begin{array}{llll}4.3 & 1.00 & -0.7 & 0.7\end{array}$

$\begin{array}{llll}3.1 & 1.13 & 0.4 & -0.3\end{array}$

$\begin{array}{llll}3.0 & 1.18 & 0.3 & -0.4\end{array}$

$\begin{array}{llll}4.2 & 1.26 & 0.0 & -0.9\end{array}$

$\begin{array}{llll}3.4 & 1.30 & 0.2 & -0.7\end{array}$

$\begin{array}{llll}4.8 & 0.98 & -0.6 & -0.3\end{array}$

$\begin{array}{llll}2.8 & 1.12 & 0.6 & 0.2\end{array}$

$\begin{array}{llll}4.4 & 1.06 & -0.6 & 0.4\end{array}$

$\begin{array}{llll}4.9 & 0.95 & -1.4 & 3.3\end{array}$

$\begin{array}{llll}4.6 & 1.03 & -0.3 & -0.8\end{array}$

$\begin{array}{llll}2.2 & 1.37 & 1.1 & 0.4\end{array}$

$\begin{array}{llll}3.1 & 1.22 & 0.7 & -0.2\end{array}$

$\begin{array}{llll}4.6 & 0.82 & -0.6 & 1.6\end{array}$

$\begin{array}{rrrr}3.2 & 1.01 & 0.6 & 0.1\end{array}$

\begin{tabular}{llll}
3.9 & 1.31 & -0.1 & -0.8 \\
\hline
\end{tabular}


Tabla 2. Confiabilidad por subescalas

\begin{tabular}{lccc}
\hline \multicolumn{1}{c}{ Subescala } & $\begin{array}{c}\text { Número de } \\
\text { ítems }\end{array}$ & $\begin{array}{c}\text { Alfa de } \\
\text { Cronbach }\end{array}$ & glb \\
\hline Comportamientos & 10 & .79 & .86 \\
Empáticos & 9 & .72 & .83 \\
Ayuda & 7 & .72 & .81 \\
Altruismo & 6 & .70 & .80 \\
Compartir y Donar &
\end{tabular}

\section{Dimensionalidad}

Previamente a la realización del análisis factorial exploratorio, se calculó el coeficiente Kaiser-Meyer-Olkin (KMO) que indicó la pertinencia de realizar el análisis $(\mathrm{KMO}=.83)$. En cuando a los resultados, se obtuvo que el primer autovalor explica el $22 \%$ de la varianza total, menor al $40 \%$ correspondiente al criterio de Carmines y Zeller (1979). La razón del primer autovalor respecto al segundo es 3.2, que es menor al 5, por lo tanto no cumple con el criterio de Martínez Arias (1995). Tampoco se cumple con el criterio de Kaiser, por existir más de un autovalor superior a 1 , ni el criterio de caída de Catell, dado que existe un salto entre el primer autovalor y los restantes, pero no es lo suficientemente brusco. Por lo tanto con todos los criterios se rechaza la unidimensionalidad (Figura $1)$.

Como era de suponer por la definición misma del constructo, la variable Conducta Prosocial, tal como se la está midiendo no puede ser considerada como unidimensional. Tomando el criterio de Kaiser, es decir la cantidad de autovalores superiores a uno, podrían existir hasta ocho dimensiones. Sin embargo, no se considera dicho criterio como decisivo, sino el criterio de caída de Cattell, ya que la regla de Kaiser ha dado lugar a sobredimensionar la solución factorial cuando, como en este caso, las variables son ítems, ya sean dicotómicos o politómicos (Navarro Loli \& Merino Soto, 2010; Ruiz \& San Martín, 1992). El diagrama de autovalores es confiable en general para determinar cuántos factores deben ser interpretados (Pérez \& Medrano, 2010). Se basa en localizar gráficamente en el diagrama de autovalores un punto en que los mismos dejan de formar una pendiente pronunciada y comienzan a generar una caída de poca inclinación (Pérez \& Medrano, 2010). En base a este criterio, se reconocen cuatro dimensiones que explican el $37,71 \%$ de la varianza.

Cada factor posee al menos cuatro ítems con correlaciones iguales o superiores a .40, lo que permite considerarlo como tal. Atendiendo a las correlaciones ítem-factor más elevadas, se observa que el primer factor presenta correlaciones más elevadas con ítems tales como: Elogio a quien se lo merece, Destaco a quienes hacen las cosas bien e Intento "levantar" la autoestima a mis amigos, es decir comportamientos verbales de refuerzo y soporte emocional (factor CE). Por otra parte, el segundo factor presenta correlaciones elevadas con ítems tales como: Participo en actividades solidarias, Aunque lo podría vender, dono lo que no uso o no me sirve y Dedico un tiempo importante de mi vida a mejorar el mundo por lo cual podría interpretarse que este factor reúne conductas tradicionales de ayuda al prójimo (factor AY). El tercer factor presenta las correlaciones más elevadas con los ítems Apoyo los paros por causas que me parecen justas aunque los mismos me perjudiquen y Expreso abiertamente mi apoyo a las minorías discriminadas aunque no pertenezca a las mismas (factor AL). En estos reactivos se expresa la anteposición de una causa noble al beneficio personal. Por último, el cuarto factor presenta las correlaciones más elevadas con los ítems Comparto con mis compañeros las experiencias que tuve en las materias que ya cursé y Explico a mis compañeros cómo hacer los trámites de la facultad. Estos ítems expresan compañerismo (factor CD).

Tabla 3. Matriz de correlaciones inter-factor

\begin{tabular}{ccccc}
\hline Subescala & $\begin{array}{c}\text { Comportamientos } \\
\text { Empáticos }\end{array}$ & Ayuda & Altruismo & $\begin{array}{c}\text { Compartir } \\
\text { y Donar }\end{array}$ \\
\hline Comportamientos & 1.00 & & & \\
Empáticos & .35 & 1.00 & & \\
Ayuda & .39 & .42 & 1.00 & \\
Altruismo & .22 & .31 & .28 & 1.00 \\
Compartir y Donar & &
\end{tabular}


Figura 1. Diagrama de autovalores

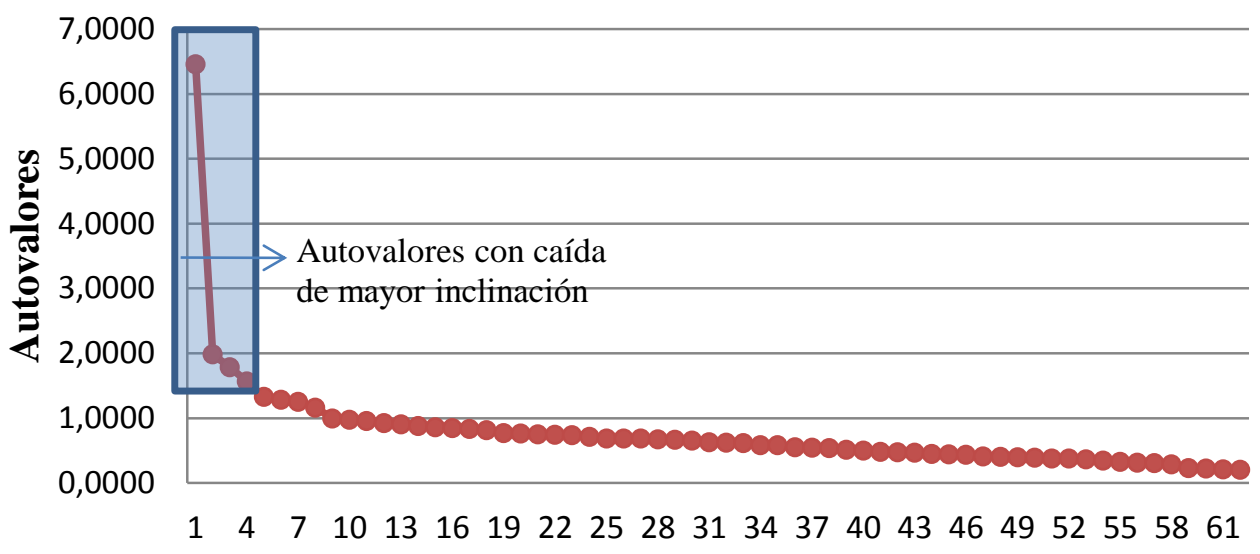

\section{Eliminación de ítems}

Se eliminaron 30 ítems de los 62 administrados en base a los criterios a) b) c) y d) expuestos. El criterio usado como base para descartar un reactivo fue el b) es decir mostrar una saturación factorial de la solución sin rotar con su factor de mayor carga menor a .35 (Cohen \& Manion, 2002), verificándose que aquellos ítems que cumplían este criterio, asimismo satisfacían los requisitos a) c) o d), es decir que mostraban una pobre calidad psicométrica en general.

Por ejemplo, el ítem No me arriesgo para defender a alguien que corre peligro fue eliminado ya que tuvo una correlación ítem-test corregida de sólo.12 mientras que las correlaciones con los factores fueron de $.20 \mathrm{con}$ el primer factor (CE), .09 con el segundo factor (AY), .13 con el tercer factor (CO) y .24 con el cuarto factor (AL). Es decir, que las puntuaciones de dicho ítem no se asocian lineal y positivamente con el puntaje total de la prueba ni con el puntaje en alguno de los factores. El ítem Si puedo hacerlo, dono sangre para desconocidos también fue eliminado ya que tuvo una correlación ítemtest corregida de sólo .13 mientras que las correlaciones con los factores fueron de $.26 \mathrm{con}$ el primer factor (CE), -.09 con el segundo factor (AY), -.17 con el tercer factor (CO) y .16 con el cuarto factor (AL). Asimismo, al quitar estos ítems aumenta levemente el Alfa de Cronbach del factor correspondiente.

\section{Discusión}

Este trabajo presenta una primera versión de la escala de Conducta Prosocialpara adultos en población universitaria argentina como una aproximación al estudio de este complejo constructo. Los análisis psicométricos del estudio realizado señalan la posible adecuación de la escala.

Los hallazgos obtenidos permitieron seleccionar 32 ítems con adecuadas propiedades psicométricas que formarán parte, en primera instancia, de una nueva Escala para la medición de la Conducta Prosocial en la etapa adulta.

La eliminación de ítems con calidad psicométrica pobre fue el principal objetivo a cumplir en esta investigación. En la prueba resultante construida a partir de los reactivos que resistieron la depuración primaria efectuada, se realizarán nuevos estudios de confiabilidad y dimensionalidad, además de estudiarse su validez convergente correlacionando su puntaje con otras pruebas que midan Conducta Prosocial.

Es de destacar que muchas conductas vinculadas con el refuerzo y soporte emocional acompañar, felicitar, escuchar- son las que exhiben un puntaje alto. La media alta en ítems de soporte emocional es muy representativa, mostrando los sujetos una uniformidad en la descripción de su comportamiento en esos reactivos. Por otra parte, ciertos ítems redactados en sentido negativo parecieran generar una reacción adversa muy fuerte, por la cual los participantes no se muestran identificados con el reactivo, lo cual es expresado con opciones 
extremas de la escala. Por ejemplo, en dos de estos ítems en que el beneficiario es un amigo, los respondientes acentúan que no sentirían que intentan cargarlos con sus problemas. En otro ítem expresan que nunca sienten satisfacción al observar los defectos ajenos.

Se observa entonces, un bajo puntaje en los ítems que reflejan los comportamientos más típicamente benéficos, tales como voluntariado, donación de ropa, sangre, comida o dinero y en algunos de los reactivos que expresan comportamientos altruistas extremos. Estos comportamientos de donación y voluntariado presentan, mayormente, un comportamiento variable, por lo que su media pierde representatividad. Además el porcentaje de los individuos que mantiene estos comportamientos es bajo.

En cuanto a la dimensionalidad, se observa una correspondencia con los factores postulados luego de la revisión teórica: "Comportamientos Empáticos" (CE), "Altruismo" (AL), "Ayuda" (AY) y "Compartir" (CD). En resumen, respecto a las propiedades psicométricas de la Escala de Conducta Prosocial, el análisis resulta coherente con el modelo planteado, acompañándose por una adecuada confiabilidad. Se destaca la posibilidad de interpretación psicológica de los resultados obtenidos en el análisis factorial exploratorio, lo cual permitirá avanzar en la construcción de diversos subtest que compondrán el test global de Conducta Prosocial.

La dimensión Comportamientos Empáticos, resultó la más fuerte, quedando con mayor cantidad de ítems posteriormente a la depuración (10 ítems) y explicando el mayor porcentaje de varianza (22\%). Es conocido que la Empatía es un componente fundamental de la Conducta Prosocial. En este sentido, Caprara et al. (2005) destacan su carácter de dimensión y la necesidad de incluirla en los test de Conducta Prosocial para adultos.

Futuras investigaciones podrán dar cuenta de si estos resultados se mantienen en estudiantes de carreras no afines a la Psicología, donde la representación de la profesión y la identificación con la misma podrían estar influyendo en las respuestas. Se buscará extender la muestra incorporando estudiantes pertenecientes a otras carreras, tanto humanísticas como de ciencias, técnicas y artísticas. Una muestra más heterogénea permitirá potenciar notablemente las posibilidades de generalización de los resultados, además se podrán realizar comparaciones de interés. También en futuras investigaciones se modelizará la Escala con Teoría de la Respuesta al Ítem (TRI) y con los Modelos Politómicos de la TRI. El comportamiento de estos últimos puede verse modulado por la cantidadde categorías de la escala Likert que se utiliza, por lo cual convendría estudiar dicha variación.

\section{Referencias}

Abal, F., Lozzia, G., Blum, G. D., Galibert, M. S., Aguerri, M. E., \& Attorresi, H. (2010). Análisis de ítems de un test de altruismo a partir del modelo logístico de un parámetro. Perspectivas en Psicología, 7(1), 16-23.

Alessandri, G., Caprara, G. V., Eisenberg, N., \& Steca, P. (2009). Reciprocal relations among self-efficacy beliefs and prosociality across time. Journal of Personality, 77, 1229 -1259. doi:10.1111/j.1467- 6494.2009.00580.x

American Educational Research Association, American Psychological Association \& National Council on Measurement in Education (1999). Standards for educational and psychological testing. Washington: American Psychological Association.

Attorresi, H., Abal, F., Lozzia, G., Picón Janeiro, J., Aguerri, M. E., \& Galibert, M. S. (2008). Construcción de una escala para la medición del Altruismo. Investigando en Psicología, 10, 7-24.

Auné, S., Blum, G. D., Abal, F., Lozzia, G., \& Attorresi, H. (2014). La Conducta Prosocial: estado actual de la investigación. Perspectivas en Psicología, 11(2), 21-33.

Batson, C. D., \& Powell, A. (2003). Altruism and prosocial behavior. En M. Theodore (Ed.) and L. Melvin (Ed.) Handbook of Psychology: Personality and Social Psychology, (5). Nueva York: John Wiley y Sons, Inc. XIX.

Bendermacher, N. (2010). Beyond Alfa: Lower Bounds for the Reliability of Tests. Journal of Modern Applied Statistical Methods, 9(1), 95102. 
Bravo, C. C., \& Lagunes, I. R. (2009). Aproximaciones metodológicas en la medición de la conducta prosocial en niños de edad escolar. Revista Iberoamericana de Diagnóstico y Evaluación - e Avaliação Psicológica, 27(1), 29-44.

Brown, T. A. (2006). Confirmatory factor analysis for applied research. New York: The Guilford Press.

Caprara, G. V., Alessandri, A., Di Giunta, L., Panerai, L., \& Eisenberg, N. (2010). The contribution of agreeableness and selfefficacy beliefs to prosociality. European Journal of Personality, 24, 36-55. doi: 10.1002/per.739

Caprara, G. V., Alessandri, G., \& Eisenberg, N. (2012). Prosociality: The contribution of traits, values, and self-efficacy beliefs. Journal of personality and social psychology, 102(6), 1289-1303. doi: 10.1037/a0025626

Caprara, G. V., Steca, P., Zelli, A., \& Capanna, C. (2005). A new scale for measuring adults prosocialness. European Journal of Psychological Assessment, 21(2), 77-89. doi: 10.1027/1015-5759.21.2.77

Carmines, E. G., \& Zeller, R. A. (1979). Reliability and validity assessment. Londres: Sage.

Cohen, L., \& Manion, L. (2002). Métodos de investigación cuantitativa. Madrid: La Muralla.

Cohen, R. J., \& Swerdlik, M. E. (2006). Pruebas y evaluación psicológicas. México: McGrawHill.

Compas, B., Davis, D., Forsythe, B., \& Warner, T. (1987). Assesment of major and daily stress full events during adolescence. The Adolescent Perceived Events Scale. Journal of Counsulting and Clinical Psychology, 55(4), 534-541.

Cronbach, L. J. (1972). Fundamentos de la exploración psicológica. Madrid: Biblioteca Nueva.

DeVellis, R. F. (2003). Scale development. Theory and applications. Thousand Oaks, CA: Sage Publications.

Eisenberg, N., Cumberland, A., Guthrie, I. K., Murphy, B. C., \& Shepard, S. A. (2005). Age changes in prosocial responding and moral reasoning in adolescence and early adulthood. Journal of Research on Adolescence, 15(3), 235-260.doi:10.1111/j.1532795.2005.00095.x

Eisenberg, N., Fabes, R. A., \& Spinrad, T. L. (2006). Prosocial development. En W. Damon \& N. Eisenberg (Eds.), Handbook of Child Psychology: Social, Emotional and Personality Development (Vol. 3, pp. 646718). Nueva York: John Wiley \& Sons.

Fisher, R. J. (1993). Social desirability bias and the validity of indirect questioning. Journal of Consumer Research, 20(2), 303-315.

García-Cueto, E., \& Fidalgo, A. M. (2005). Análisis de los ítems. En J. Muñiz, A.M. Fidalgo, E. García-Cueto, R. Martínez y R. Moreno (Eds.), Análisis de los ítems (pp. 53130). Madrid: La Muralla.

Gempp, R., Denegri, M., Caripán, N., Catalán, V., Hermosilla, S., \& Caprile, C. (2007). Desarrollo del test de alfabetización económica para adultos. Interamerican Journal of Psychology, 41(3), 275-284.

George, D., \& Mallery, M. (2003). Using SPSS for Windows step by step: a simple guide and reference. Boston, MA: Allyn y Bacon.

Gil-Escudero, G., \& Martínez-Arias, M. R. (2001). Metodología de encuestas. En M. J. Navas (Ed.), Métodos, diseños y técnicas de investigación psicológica (pp. 379 -436). Madrid: Universidad Nacional de Educación a Distancia.

Glutting, J. J., Monaghan, M. C., Adams, W., \& Sheslow, D. (2002). Some psychometric properties of a system to measure ADHD among college students: Factor pattern, reliability, and one-year predictive validity. Measurement and Evaluation in Counseling and Development, 34, 194 - 208.

González Portal, M. D. (2000). Conducta Prosocial: Evaluación e Intervención. Madrid: Morata.

Hay, D. F., \& Cook, K. V. (2007). The transformation of prosocial behavior from infancy to childhood.En C. A. Brownell \& C. B. Kopp (Eds.), Socioemotional development in the toddler years: Transitions and transformations (pp. 100-131). New York: The Guilford Press. 
Inglés, C. J., Martínez-González, A. E., Valle, A., García-Fernández, J. M., \& Ruiz-Esteban, C. (2010). Conducta Prosocial y motivación académica en estudiantes españoles de Educación Secundaria Obligatoria. Universitas Psychologica, 10(2), 451-465. doi: $10.11144 / 756$

Kaiser, H. F. (1960). The application of electronic computers to factor analysis. Educational and Psychological Measurement, 20, 141-151. doi: 10.1177/001316446002000116

López de Cózar, E., Alonso Plá, F., Esteban Martínez, C., Calatayud Miñana, C., \& Alamar Rocati, B. (2008). Diseño y validación de una escala para la medición de la Conducta Prosocial - antisocial en el ámbito vital y en el tráfico. Instituto Universitario de Tráfico y Seguridad Vial. Universidad de Valencia.

Lorenzo-Seva, U., \& Ferrando, P. J. (2006). FACTOR: A computer program to fit the exploratory factor analysis model. Behavioral Research Methods, Instruments and Computers, 38(1), 88-91. doi: 10.3758/BF03192753

Luengo, M. A., Otero, J. M., Romero, E., GómezFraguela, J. A., \& Tavares-Filho, E. T. (1999). Análisis de ítems para la evaluación de la conducta antisocial: un estudio transcultural. Revista Iberoamericana de Diagnóstico y Evaluación - e Avaliação Psicológica, 1, 2136.

Martínez Arias, R. (1995). Psicometría: Teoría de los Tests Psicológicos y Educativos. Madrid: Síntesis.

Navarro Loli, J., \& Merino Soto, C. (2010). Un réquiem para la regla de Kaiser (eigen> 1 ) en la retención del número de factores. Fractal Revista de Psicología, 22(3), 641-642.

Pérez, E., \& Medrano, L. A. (2010). Análisis factorial exploratorio: Bases conceptuales y metodológicas. Revista Argentina de Ciencias del Comportamiento, 2(1), 58-66.

Ruiz, M. A., \& San Martín, R. (1992). Una simulación sobre el comportamiento de la regla $\mathrm{K} 1$ en la estimación del número de factores. Psicothema, 4(2), 543-550.
Tornimbeni, S., Pérez, E., \& Olaz, F. (2008). Introducción a la psicometría. Buenos Aires: Paidós.

Waller, N. G. (2001). MicroFACT 2.0: A microcomputer factor analysis program for ordered polytomous data and mainframe size problems. Assessment Systems Corporation, USA.

Warneken, F., \& Tomasello, M. (2009).The roots of human altruism. British Journal of Psychology, 100(3), 455-471.doi: $10.1348 / 000712608 \times 379061$ 